\title{
Introduction Spinal Deformity
}

\author{
January 2003
}

By J. PATRick Johnson, M.D., ANd Christopher I. ShafFrey, M.D.

In this issue of Neurosurgical Focus topic we focus on the highly diverse discipline of spinal deformity. This has become a subspecialty of spinal surgery that is even further categorized into pediatric and adult subspecialties. Articles written by the recognized authorities and the future leaders in spinal deformity treatment are presented in this issue. The issue also reflects a unique collaboration between neurosurgical and orthopedic colleagues who have coauthored many of these papers. We hope this topic stimulates your interest and ultimately leads to improved patient outcomes.

The treatment of spinal disorders continues to evolve at a pace that defies prediction. The concepts of spinal deformity with regard to biomechanics and spinal alignment are now recognized as essential aspects in the management of spinal disorders. The terms sagittal- and coronal-plane balance of the spinal column are used in daily clinical practice to signify the direct effects on adjacent spinal segments and the entire spinal column, such balance is essential for optimum outcomes. Spinal instrumentation has evolved from crude to highly sophisticated devices but still has significant shortcomings. In the past, treatments often involved brace therapy and surgical correction, which achieved only minimum correction of the deformity or in situ fusion that simply prevented its progression. Currently segmental instrumentation and fixation techniques allow the manipulation, correction, and maintenance of severe deformities with remarkable precision and safety.

In the future, correction of spinal deformity may include biological enhancement of spinal fusion, dynamic spinal instrumentation that can be preprogrammed and reprogrammed to achieve a desired outcome, and novel uses of tissue generation and regeneration. Other functional spinal reconstruction technology involving artificial disc replacement is beginning to emerge and will likely affect the treatment of spinal deformity.

Neurosurg. Focus / Volume 14 / January, 2003 